\title{
A Method of Determining Realistic Stress S/N Curves by Interpolations and Extrapolations of Two Known Best-Fit S/N Curves for Fatigue Life Predictions
}

\author{
Claudiu Gudas \\ C \& D Gudas, Sydney, Australia \\ Email: Claudiu.Gudas@bigpond.com
}

How to cite this paper: Gudas, C. (2018) A Method of Determining Realistic Stress S/N Curves by Interpolations and Extrapolations of Two Known Best-Fit S/N Curves for Fatigue Life Predictions. Materials Sciences and Applications, 9, 368-380. https://doi.org/10.4236/msa.2018.94025

Received: February 10, 2018

Accepted: April 15, 2018

Published: April 18, 2018

Copyright $\odot 2018$ by author and Scientific Research Publishing Inc. This work is licensed under the Creative Commons Attribution International License (CC BY 4.0).

http://creativecommons.org/licenses/by/4.0/ (c) (i) Open Access

\begin{abstract}
The design and sizing of new mechanical components which are intended to operate under cyclic loads often require an acceptable level of confidence that the components will meet pre-defined fatigue strength objectives for crack initiation. For loads with multiple amplitudes and mean values, models based on Palmgren-Miner's linear cumulative damage hypothesis and on multiple $\mathrm{S} / \mathrm{N}$ curves (stress-no. cycles) are widely used in estimations for the duration of crack initiation. In this paper a procedure for generating $\mathrm{S} / \mathrm{N}$ curves for multiple stress ratios by interpolation or extrapolation from the data available for two such curves is proposed. At any number of cycles, the stress for crack initiation is calculated from the far field macroscopic stresses of the known curves using Dang Van fatigue criterion and microscopic stresses evaluated at the grain level. An algorithm is presented for uniaxial loading and results verifications against curves established from laboratory tests with elastic and plastic stresses are shown and discussed for notched and un-notched specimens.
\end{abstract}

\section{Keywords}

Crack Initiation, Extrapolation, Fatigue Life, Interpolation, Stress Ratio, The Dang Van Criterion

\section{Introduction}

With the rise of fracture mechanics, the certification against fatigue has largely switched towards crack-propagation models which correlate parameters like the crack tip stress intensity factor to its growth rate. At the same time new stronger 
materials have led to higher working stress and, in many situations, having higher crack growth rates, thus once initiated the duration of crack growth to a critical size is reduced [1]. Many commercial software packages dedicated to crack growth analysis incorporate modules for estimation of crack initiation. These modules are using strain-life initiation methodologies like Smith-WatsonTopper and require stabilized cyclic stress-strain data [2]. Crack growth analyses are significantly more laborious than traditional fatigue analyses. Often they can be done efficiently only after the design is finalized. In these situations also, the ability to estimate early from a traditional fatigue life approach the duration for crack initiation and growth to a predefined size is very beneficial during early design stages.

In the traditional approaches to fatigue strength evaluation for loading spectra containing cycles with multiple maximum and mean stress values, the degree of cumulative damage at each stress level is calculated from $\mathrm{S} / \mathrm{N}$ curves. There are many forms in which these curves could be presented and most of them are obtained from uni-axial cupon tests after statistical post-processing of the results to account for scatter. For uni-axial tests, all these forms can be converted to express the cycles in terms of maximum stress and stress ratio $(R)$. In case of multiaxial loading, more parameters are needed to specify the phase characteristics or the principal stresses and angles. The failure criteria used during testing can represent either the crack initiation, when the length of the crack is about 1.0 $\mathrm{mm}$ or 0.040 inch or complete fracture. In this approach only curves of first criteria are considered.

Due to its simplicity and larger availability of uni-axial S/N data, the evaluation of crack initiation using Miner's rule is a pragmatic and quick alternative to other stress based methods like the critical plane approach, Crosslands, Sines.

In practice however, during and sizing, it is frequent that the data available is for a limited number of stress ratios which often do not cover the range of stresses required. The method presented below enables generation of $\mathrm{S} / \mathrm{N}$ curves for new stress ratios from two known curves of stress ratios $R_{1}$ and $R_{2}$.

\section{Method Outline and Verification Details}

\subsection{Brief Overview of Dang Van Fatigue Criterion}

During fatigue testing of materials, when the peak stresses exceed the yield limit they generate plastic flow and residual stresses [3]. At the same time, due to Bauschinger effects, after a certain amount of plastic deformation in one direction, the material yields at a lower level in the opposite direction. Within several cycles the stress-strain variations stabilize to a shake down state where the residual stresses alleviate the applied loads and induce a state of elastic response. In an elastic or shacked-down state no fatigue damage is supposed to occur because of no further strain accumulations. Tests however clearly demonstrate that fatigue occurs during cycles with peak stresses less than half of the material's yield values. 
Dang Van took the above concept to the grain level and noted that plasticity at this level can exist even when the macroscopic stresses are in the elastic range. He noted that a crack will initiate when the microscopic shake-down limits are just exceeded (called it a pseudo shake-down state) [4] [5] [6].

A brief outline of the mathematical formulation for estimating the microscopic stresses:

- The macroscopic stress tensor $\underline{\Sigma}$ is expressed in terms of hydrostatic pressure $P H$ and the deviatoric $\underline{S}$ tensor;

- Similarly, the microscopic stress tensor $\underline{\sigma}$, is formulated in terms of microscopic hydrostatic pressure and deviatoric tensor, $p h$ and $\underline{s}$, respectively;

- The relations between the macro and microscopic stresses:

o the hydrostatic pressure is the same at macro and microscopic levels $(\mathrm{PH}=$ ph);

o the macroscopic deviatoric stresses are given by the sum of the microscopic deviatoric and stabilized residual stresses $q$;

It was postulated in [4] that the stabilized residual stresses are at the center of the smallest hypersphere in the six-dimensional deviatoric stress space that completely encloses the load path. Tensor $q$ needs to include only the deviatoric stresses $q^{*}$, as only these stresses affect sliding along intergranular preferential slip bands (PSB) and the initiation of shear induced cracks.

$$
\underline{S}=\underline{s}+\underline{q}^{*}
$$

Noting that both, the hydrostatic pressure $p h$ and maximum shear $\sigma_{\max }$ of the microscopic stresses affect crack initiation, Dang Van et al. [2] formulated a fatigue crack criterion in the $p h-\tau$ space as being defined by the two lines $D$ and $D^{\prime}$ shown in Figure 1. The lines are given by Equation (2), where the coefficients a and $b$ are material dependent real constants.

$$
\tau_{\min / \max }= \pm a * p h+b,
$$

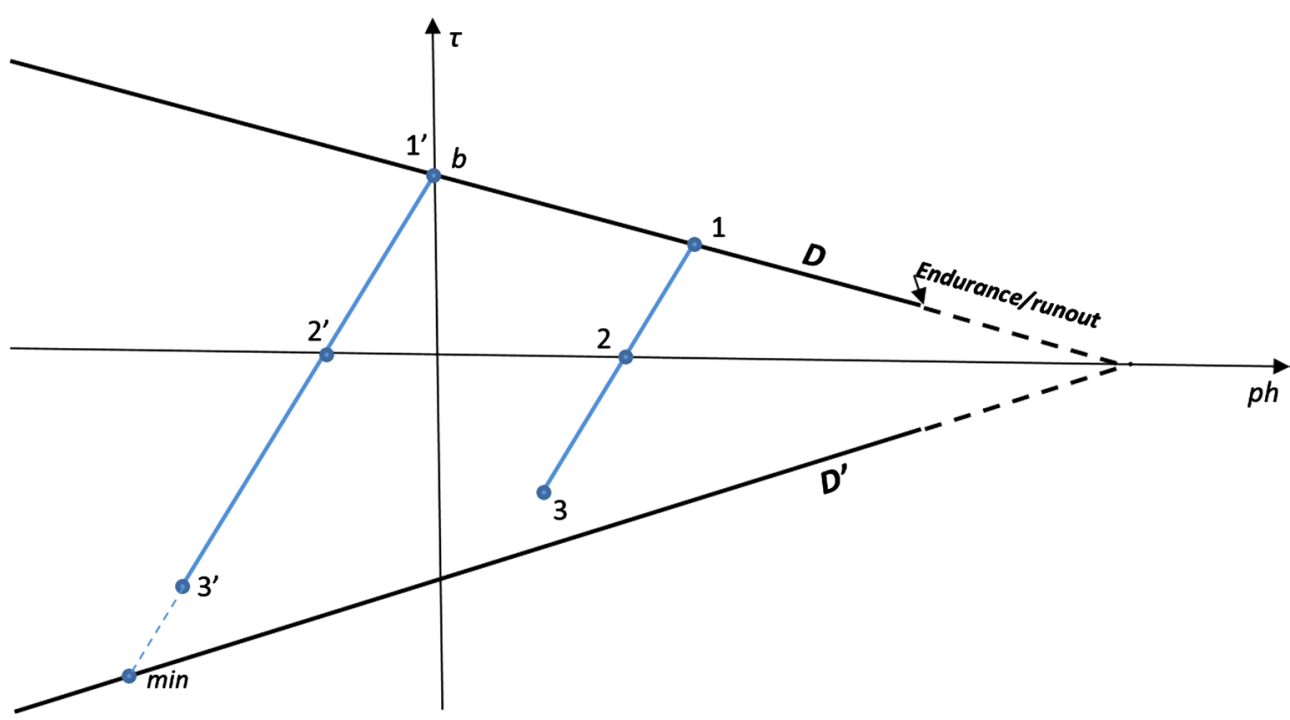

Figure 1. Fatigue initiation lines in the microscopic $p h-\tau$ space. 
and:

$$
\left|\tau_{\min }\right|=\left|\tau_{\max }\right|=\operatorname{Tresca}(s) .
$$

Test verifications of this criterion show that coefficients $a$ and $b$ in Equation (2) remain constant with the change of stress ratios from -1.75 to +0.2 and with type of loading (shear, tension and biaxial). It is also shown that with fatigue life decrease there is a substantial decrease in $|a|$ at the same time as an increase in $b$ [5].

\subsection{Method Outline}

For any of the known uni-axial stress $\mathrm{S} / \mathrm{N}$ curves of stress ratio $R_{k} k=\{1,2\}$, the temporal variation of the stress is assumed to be of the form:

$$
\Sigma_{k}=\left(\begin{array}{c}
\sigma_{k_{-} x} \\
\sigma_{k_{-} y} \\
\tau_{k_{-} x y}
\end{array}\right)=\left(\begin{array}{c}
\frac{A_{k}}{2} *\left[\frac{1+R_{k}}{1-R_{k}}+\sin \left(\omega_{k} t\right)\right] \\
0 \\
0
\end{array}\right),
$$

where the amplitude of cyclic stresses $A_{k}$ is defined by:

$$
A_{k}=\frac{2 * \sigma_{k_{-} \max }}{\frac{1+R_{k}}{1-R_{k}}+1} \text {, if } R_{k}<1 \text { and } A_{k}=\frac{2 * \sigma_{k_{-} \max }}{\frac{1+R_{k}}{1-R_{k}}-1} \text {, if } R_{k}>1
$$

The macro and microscopic hydrostatic pressures at the measurement locations are:

$$
\begin{aligned}
P H_{k}(t) & =p h_{k}(t) \\
& =\frac{A_{k}}{6} *\left[\frac{1+R_{k}}{1-R_{k}}+\sin \left(\omega_{k} t\right)\right],
\end{aligned}
$$

and the macroscopic deviatoric stresses,

$$
\left(\begin{array}{l}
S_{k_{-} 1} \\
S_{k_{-} 2} \\
S_{k_{-} 3}
\end{array}\right)=\left[\frac{1+R_{k}}{1-R_{k}}+\sin \left(\omega_{k} t\right)\right] *\left(\begin{array}{c}
\frac{A_{k}}{3} \\
\frac{-A_{k}}{6} \\
\frac{-A_{k}}{6}
\end{array}\right)
$$

For uniaxial loading the load path is a straight line and the deviatoric of the microscopic residual stress tensor is:

$$
\left(\begin{array}{c}
q_{k_{-} 1}^{*} \\
q_{k_{-} 2}^{*} \\
q_{k_{-} 3}^{*}
\end{array}\right)=\frac{1+R_{k}}{1-R_{k}} *\left(\begin{array}{c}
\frac{-A_{k}}{3} \\
\frac{A_{k}}{6} \\
\frac{A_{k}}{6}
\end{array}\right)
$$

From Equation (1) the microscopic deviatoric stresses are: 


$$
\left(\begin{array}{c}
s_{k_{-} 1} \\
s_{k_{-} 2} \\
s_{k_{-} 3}
\end{array}\right)=\sin \left(\omega_{k} t\right) *\left(\begin{array}{c}
\frac{A_{k}}{3} \\
\frac{-A_{k}}{6} \\
\frac{-A_{k}}{6}
\end{array}\right)
$$

The resulting maximum microscopic shear is

$$
\tau_{k_{\_} \max }=\operatorname{Tresca}\left(s_{k}\right)=\left|\frac{A_{k}}{4} * \sin \left(\omega_{k} t\right)\right|, \quad k=\{1,2\}
$$

With $\left(p h_{1}, \tau_{1 \_\max }\right)$ and $\left(p h_{2}, \tau_{2 \_ \text {max }}\right)$ calculated for the same life $N_{f}$ the values of $a$ and $b$ defining the lines for the Dang Van fatigue criterion at $N_{f}$ are given by Equation (2).

From Equation (5), for a life of $N_{f}$ cycles, the amplitude of cycles of other stress ratio $R$ is given by:

$$
A=\frac{b}{\frac{1}{4}-\frac{a}{6} *\left(\frac{1+R}{1-R}+1\right)}, \text { if } R_{k}<1 \text { and } A=\frac{b}{\frac{1}{4}-\frac{a}{6} *\left(\frac{1+R}{1-R}-1\right)}, \text { if } R_{k}>1
$$

Based on the study in reference [7] the equations above were assessed as suitable for finite lifetimes and for plastic macroscopic stresses.

Referring to Figure 1, for any cycle of stress ratios $R$, the points 1, 2 and 3 defined by Equation 6 and 10 when $\sin (\omega t)$ takes the values of 1, 0 and -1 , respectively are:

- Point 1 of coordinates $p h_{1}=\frac{A}{6} *\left[\frac{1+R}{1-R}+1\right]$ and $\tau_{\max 1}=\frac{A}{4}$;

- Point 2 of coordinates $p h_{2}=\frac{A}{6} *\left[\frac{1+R}{1-R}\right]$ and $\tau_{\max 2}=0$;

- Point 3 of coordinates $p h_{3}=\frac{A}{6} *\left[\frac{1+R}{1-R}-1\right]$ and $\tau_{\max 3}=-\frac{A}{4}$.

The slopes $m_{12}$ and $m_{23}$ for segments 12 and 23 show that the three points are on the same line independent of $R$ :

$$
\begin{aligned}
& m_{12}=\frac{\tau_{\max 1}}{p h_{1}-p h_{2}}=\frac{\frac{A}{4}}{\frac{A}{6} *\left[\frac{1+R}{1-R}+1\right]-\frac{A}{6} *\left[\frac{1+R}{1-R}\right]}=\frac{6}{4}=1.5 \\
& m_{23}=\frac{-\tau_{\max 3}}{p h_{2}-p h_{3}}=\frac{\frac{A}{4}}{\frac{A}{6} *\left[\frac{1+R}{1-R}\right]-\frac{A}{6} *\left[\frac{1+R}{1-R}-1\right]}=\frac{6}{4}=1.5
\end{aligned}
$$

For the particular case when $\sigma_{\max }=0(R=\infty)$, the microscopic hydrostatic pressure and shear stress for $\sigma_{\min }$ are given by the intersection of line $D^{\prime}$ with the line 1'2'3' passing though $(0, b)$ :

$$
\tau=1.5 * p h_{\min }+b, \quad \tau=a * p h_{\min }+b
$$




\subsection{Verification Details}

The algorithm outlined above was verified using Mathcad v.14, for the materials and specimen forms indicated in Table 1. The data used for these verifications contains $\mathrm{S} / \mathrm{N}$ curves for multiple stress ratios, explicit equations for the fatigue models used and ranges of applicability. Many of the curves have stresses in plastic range enabling verifications of the method at stress levels typical for fail-safe designs.

For each test, two curves $R_{1}<R_{2}$, were selected as "known data" and discretized in three columns tables containing stress, life an $d \log _{10}($ life $)$. For each of the "new" stress ratio $R$, relative differences were calculated in terms of $\log _{10}($ life $)$ and in terms of maximum stress, using:

$$
\delta_{\text {LogLife }}(\sigma)=\frac{\log _{10}\left(n_{0}\right)-\log _{10}(n)}{\log _{10}\left(n_{0}\right)} \text { and } \delta_{\sigma}(\text { life })=\frac{\sigma_{0}-\sigma}{\sigma_{0}}
$$

$n_{0}$-life at $\sigma$, for the curve generated from published equation

$n$-life at $\sigma$, for the curve generated from two known $\mathrm{S} / \mathrm{N}$ curves

$\sigma_{0}$-stress at given life, for the curve generated from published equation

$\sigma$-stress at given life, for the curve generated from two known S/N curves

During the verifications, all tables were limited to 15 lines with constant increments in stress or in $\log _{10}$ (life). The relative differences were calculated at each entry in the output tables and checked for direction and trends. Cubic splines in stress and logarithm of life were used when additional points were required to extend the curves.

The testing process included extrapolations and interpolations. For extrapolations $R_{1}$ and $R_{2}$ were selected for two enveloping cases and for several intermediate ones.

- In the first enveloping case $R_{1}$ was selected at the lowest available stress ratio and $R_{2}$ was 0.1 higher than $R_{1}$. New curves, in increments of 0.1 , were extrapolated to the highest stress ratio in the range of applicability and compared

Table 1. Materials and configurations used for verifications.

\begin{tabular}{|c|c|c|c|c|c|c|c|c|}
\hline \multirow{2}{*}{ Material } & \multirow{2}{*}{ Notch } & \multirow{2}{*}{ Form } & \multicolumn{2}{|c|}{ Ultimate Strength } & \multicolumn{2}{|c|}{ Yield Strength } & \multirow{2}{*}{$\mathrm{SR}^{[\mathrm{b}]}$} & \multirow{2}{*}{ Figure ${ }^{[c]}$} \\
\hline & & & $\left(k s 1^{[a]}\right)$ & $(M P a)$ & $(k s i)$ & $(M P a)$ & & \\
\hline \multirow[t]{2}{*}{ 2024-T4 Alu. Alloy, L Dir. } & 1.00 & Drawn & 71 & 490 & 44 & 303 & -1.00 to 0.50 & 3.2.4.1.8(a) \\
\hline & & Extr. & 85 & 586 & 65 & 448 & & \\
\hline 2024-T4 Alu. Alloy, L Dir. & 2.40 & Bar & 73 & 503 & 49 & 338 & -1.00 to 0.74 & 3.2.4.1.8(c) \\
\hline 2024-T3 Alu. Alloy, L Dir. & 1.00 & Sheet & $72-73$ & $496-503$ & $52-54$ & $359-372$ & -1.00 to 0.50 & $3.2 .4 .1 .8(\mathrm{e})$ \\
\hline Ti-6Al-4V, Annealed, L Dir. & 2.43 & Bar & 150 & 1034 & 143 & 986 & -1.00 to 0.40 & 5.4.1.1.8(b) \\
\hline Ti-6Al-4V, Annealed, LT Dir. & 1.00 & Sheet & $147-152$ & $1014-1048$ & $136-143$ & $938-986$ & -0.50 to 0.50 & $5.4 .1 .1 .8(\mathrm{f})$ \\
\hline
\end{tabular}

${ }^{[\mathrm{a}]}$ kilo pound $/ \mathrm{in}^{2},(1 \mathrm{ksi}=6.895 \mathrm{MPa}) ;{ }^{[\mathrm{b}]}$ Range of stress ratios; ${ }^{[\mathrm{c}]}$ From reference [8]. 
with the curves generated from equations;

- In the reverse case, the two $R_{1}$ and $R_{2}$ curves were selected at the highest end and the new curves were extrapolated in a similar manner to the lowest available stress ratio;

- In the intermediate cases, like that exemplified in Figure 2, the two known curves were aleatory placed and extrapolations were conducted upwards and/or downwards.

For interpolations, the extreme envelope considered was when the two sets of known data were at the lowest and respectively the highest values of the published range of applicability. Additional tests were conducted between stress ratio of zero and the minimum or maximum available.

\section{Results}

The results for all S/N curves generated by extrapolation were conservative (safer than the results of the curves constructed from the published equations). It was observed that as the distance of extrapolation increases so does the level of conservatism. For interpolations the resulting curves are optimistic with the relative differences increasing towards the middle of interval between $R_{1}$ and $R_{2}$.

Focusing on the enveloping tests for which the relative differences have the

SN Diagrams DV Criterion vs Explicit Equation

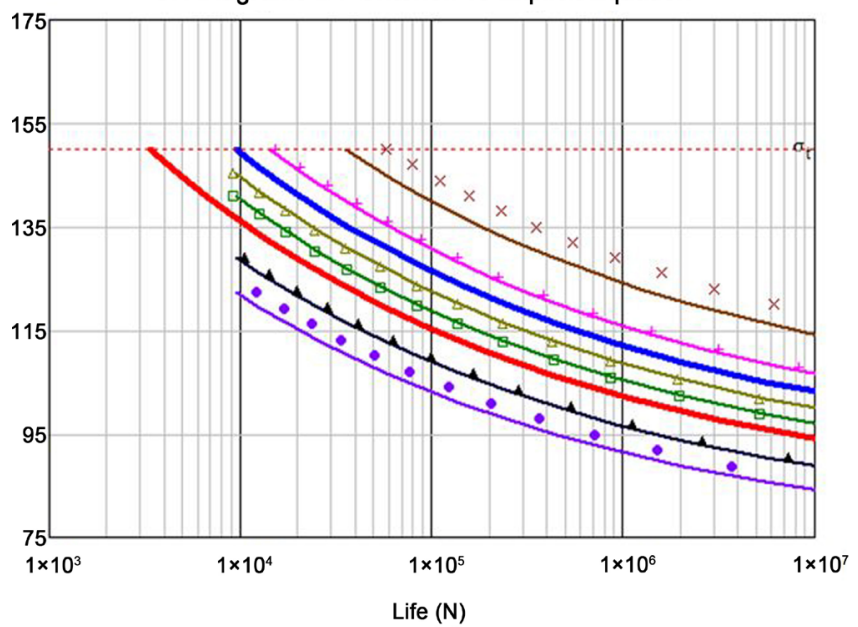

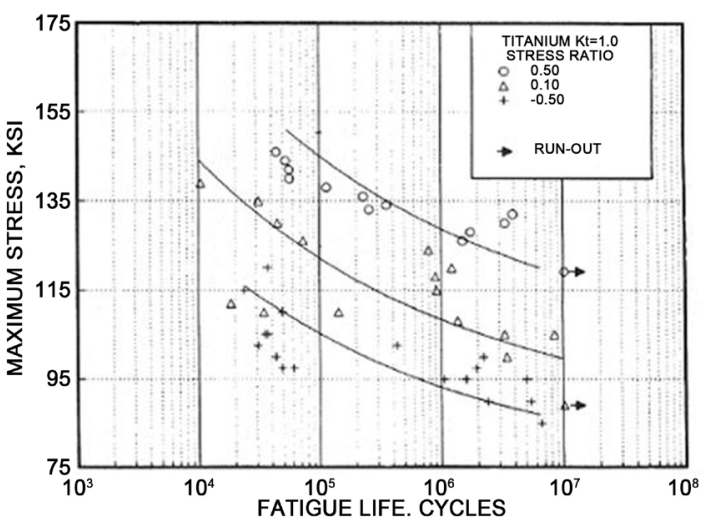

Figure 5.4.1.1.8(f). Best-fit S/N curves for unnotched TI-6AL-4V annealed sheet, long transverse direction

Correlative Information for Figure 5.4.1.1.8(f) Loading - Axial

$R_{1}=-0.1 \quad R_{2}=0.2$

$\mathrm{RR}_{1}=-0.5 \quad \mathrm{RR}_{2}=-0.3$

$\mathrm{RR}_{3}=0 \quad \mathrm{RR}_{1}=0.1$

$\mathrm{RR}_{5}=0.3 \quad \mathrm{RR}_{6}=0.5$

- New Curve RR2 DV Criterion

^4 Curve RR2 From Eq

- New Curve RR3 DV Criterion

and Curve RR3 From Eq

- New Curve RR4 DV Criterion

$\triangle \triangle \triangle$ Curve RR4 From Eq

- New Curve RR5 DV Criterion

+++ Curve RR5 From Eq

- New Curve RR6 DV Criterion

$x x \times$ Curve RR6 From Eq

(a)

Product Form: Sheet, 0.063-, 0.070-, 0.078-inch Test Parameters: Frequency -- 10-95 Hz

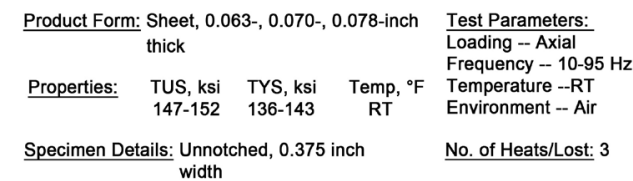

Surface Conditions: Machined to 32 RMS, lightly polished with 400 grit emery paper

Reference: $5 \cdot 4 \cdot 1.1 .8(\mathrm{c})$

Equivalent Stress Equations: $\log \mathrm{N}_{\mathrm{s}}=12.59-4.891 \log \left(\mathrm{S}_{\mathrm{aq}}-82.8\right)$ $\mathrm{Sq}=\mathrm{Smax}(1-\mathrm{R}) 0.29$ Std. Error of Estimate, Log(Life $)=0.62$ Standard Deviation, $\log ($ Life $)=0.88$ $\mathrm{R}_{2}=50.6 \%$

Sample Size $=47$

[Caution. The equivalent strain model may provide unrealistic life predictions for strain ratios and ranges beyond those represented above.]

(b)

Figure 2. Graphical sample of $\mathrm{S} / \mathrm{N}$ curves in verifications. (a) $\mathrm{S} / \mathrm{N}$ curves generated by interpolation and extrapolation from $R_{1}$ and $R_{2}$; (b) Reference data for $R_{1}, R_{2}$ and for verifications of relative differences (extract from [8]) 
largest values and using as parameter $\delta R$, defined by the distance between the new stress ratio and the closest of $R_{1}$ or $R_{2}$ :

- For extrapolations: At the left end of the curves representing the high stresses and shorter lives, both $\delta_{\sigma}$ (life) and $\delta_{\text {Loglife }}(\sigma)$ are less than $10 \%$ up to a $\delta R$ of around $0.4-0.6$ and they increase afterwards to $25 \%$ as $\delta R$ approaches 1.5 . At the other end (of low stresses and long lives) the estimates are smaller than $15 \%$ for $\delta R$ less or equal to 0.5 and increase to $30 \%$ afterwards. At this end however, the runout or the endurance limits will frequently reduce these differences.

- For interpolations: When the distance between $R_{1}$ and $R_{2}$ is less than $1.2-1.3$ the relative differences are below $8 \%$ in both measures. They increase to $15 \%$ for larger distances between $R_{1}$ and $R_{2}$.

Figure 3 shows the maximum values of $\delta_{\text {Loglife }}(\sigma)$ with the increase in $\delta R$ for the extrapolation envelopes while Figure 4 shows maximum values of $\delta_{\sigma}$ (life). When the distance between $R_{1}$ and $R_{2}$ is larger than 0.1 the maximum relative differences vs. $\delta R$ are smaller than those shown in two figures referenced above.

For interpolations the results of the enveloping cases are shown in Figure 5. Table 2 and Table 3 summarize the differences when one of the known stress ratios is zero.
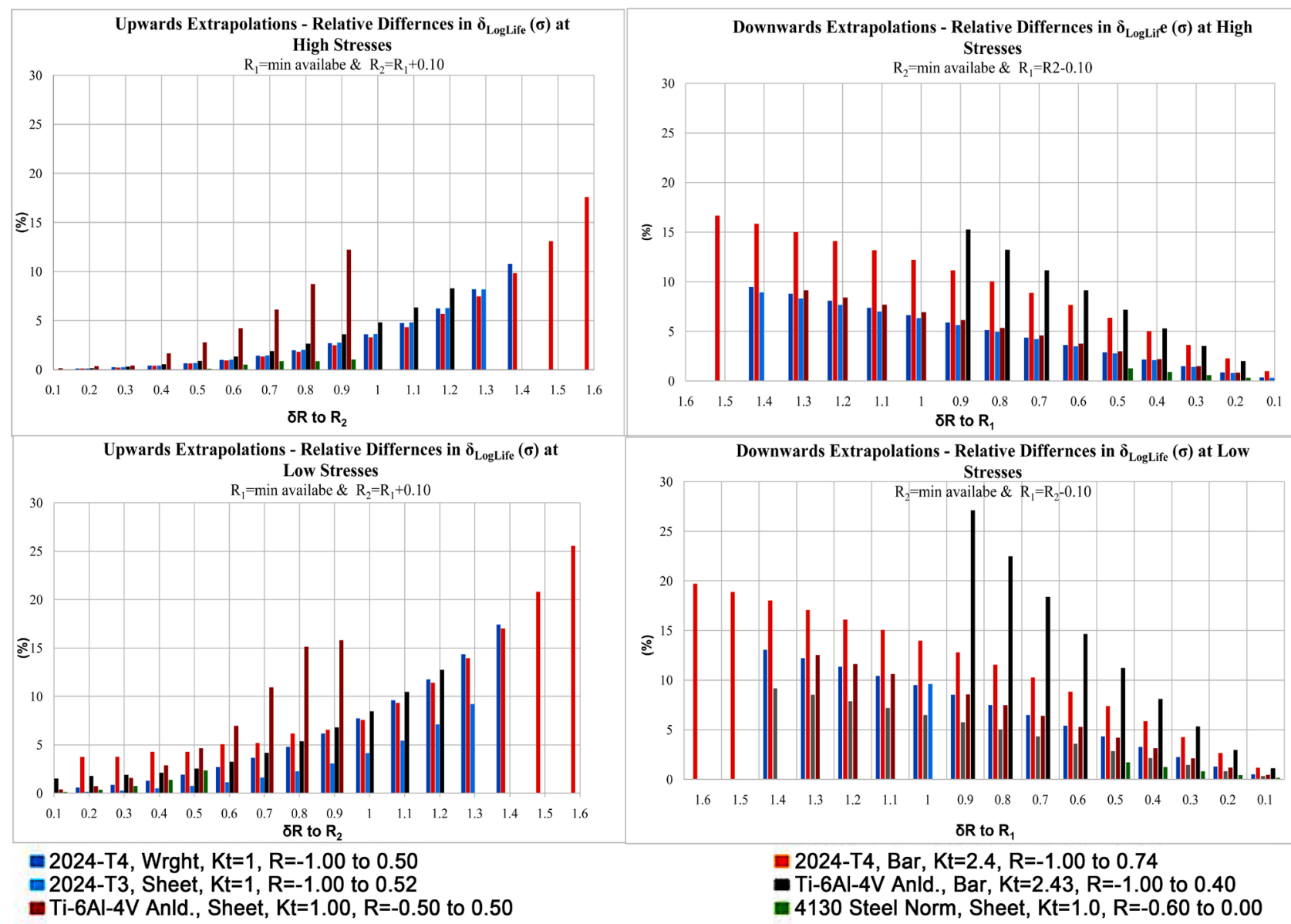

2024-T3, Sheet, $K t=1, R=-1.00$ to 0.52

Ti-6Al-4V Anld., Sheet, $\mathrm{Kt}=1.00, \mathrm{R}=-0.50$ to 0.50

4130 Steel Norm, Sheet, $K t=1.0, R=-0.60$ to 0.00

Figure 3. Extrapolations-Maximum relative differences in $\delta_{\text {LogLife }}(\sigma)$. (left) for upward extrapolations; (right) for downward extrapolations (top) for high stresses; (bottom) for low stresses. 

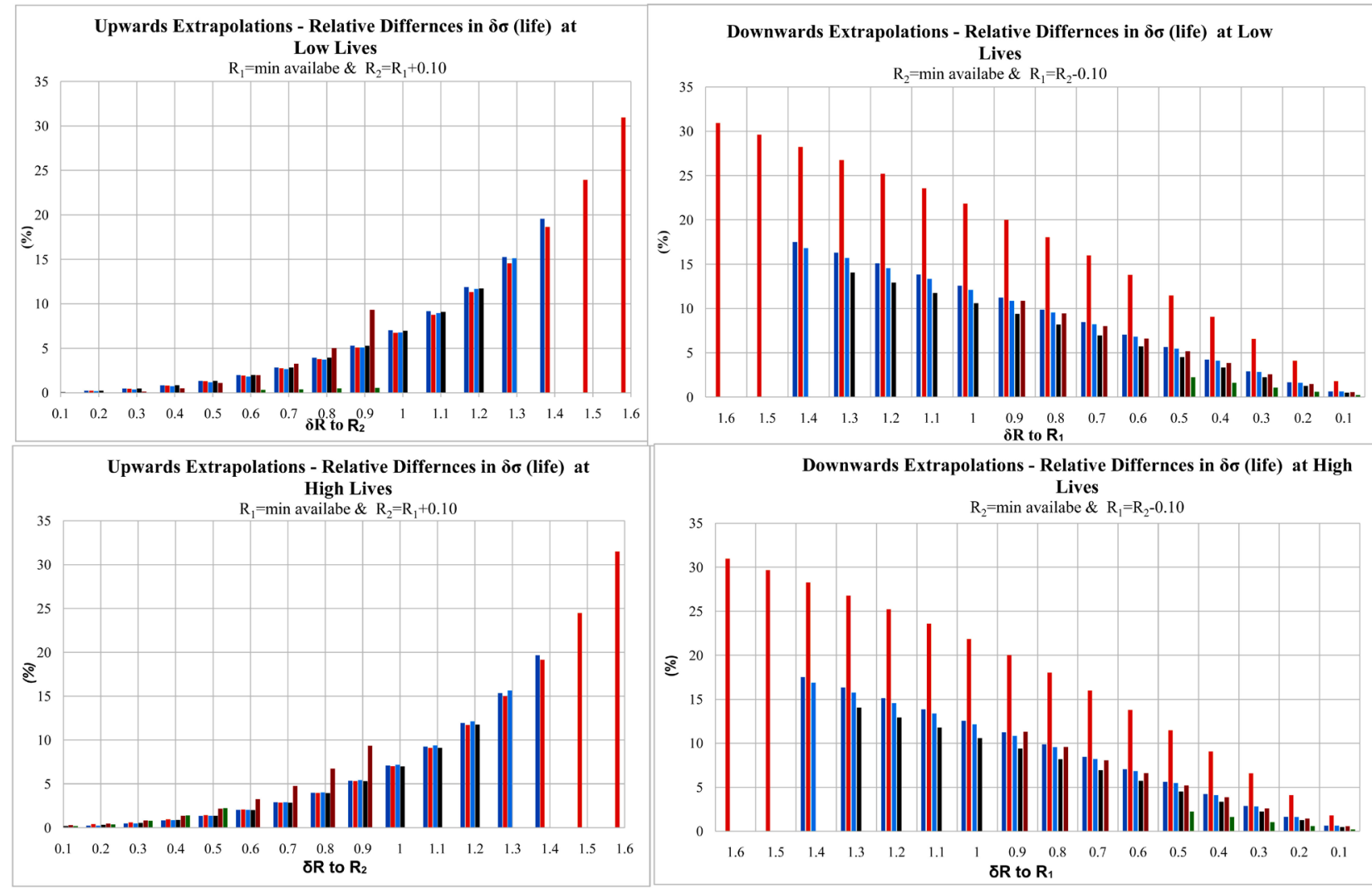

2024-T4, Wrght, $K t=1, R=-1.00$ to 0.50

- 2024-T3, Sheet, $\mathrm{Kt}=1, \mathrm{R}=-1.00$ to 0.52

$\because$ Ti-6Al-4V Anld., Sheet, Kt=1.00, R=-0.50 to 0.50

E2024-T4, Bar, $\mathrm{Kt}=2.4, \mathrm{R}=-1.00$ to 0.74

- Ti-6Al-4V Anld., Bar, $\mathrm{Kt}=2.43, \mathrm{R}=-1.00$ to 0.40

-4130 Steel Norm, Sheet, $\mathrm{Kt}=1.0, \mathrm{R}=-0.60$ to 0.00

Figure 4. Extrapolations-Maximum relative differences in $\delta_{\sigma}$ (life). (left) for upward extrapolations; (right) for downward extrapolations (top) for shot lives; (bottom) for long lives.

\section{Discussion}

The tests show that for interpolations and extrapolations with moderate $\delta R(\leq 0.5)$ the results are comparable with test data. For very large extrapolations, the curves might be too conservative from a weight or economic perspective and another curve reducing the span between the known stress ratios is required. When the known data was for high tension stress ratios, cubic splines proved to be effective and accurate in extending the new curves of lower stress ratios.

These tests also showed realistic results for notched specimens with moderate stress concentration factors (orange and black entries in Figure 3 to Figure 5). Note that for these situations the cyclic stress are specified in terms of net or gross area averages and the lines D and D' in Figure 1, are only far-away indicators for the crack initiation at the notch.

It is therefore noted that in current form the algorithm presented in Equations (1) to (12) be limited to notch specimens with a stress concentration factor not larger than 2.4 when the "known" data represents the average area stresses.

For larger concentration factors, the macroscopic stresses at the notch enter quickly in plastic range and the changes of the local values from the net or gross area far field average become significant. In these situations, for part sizing 


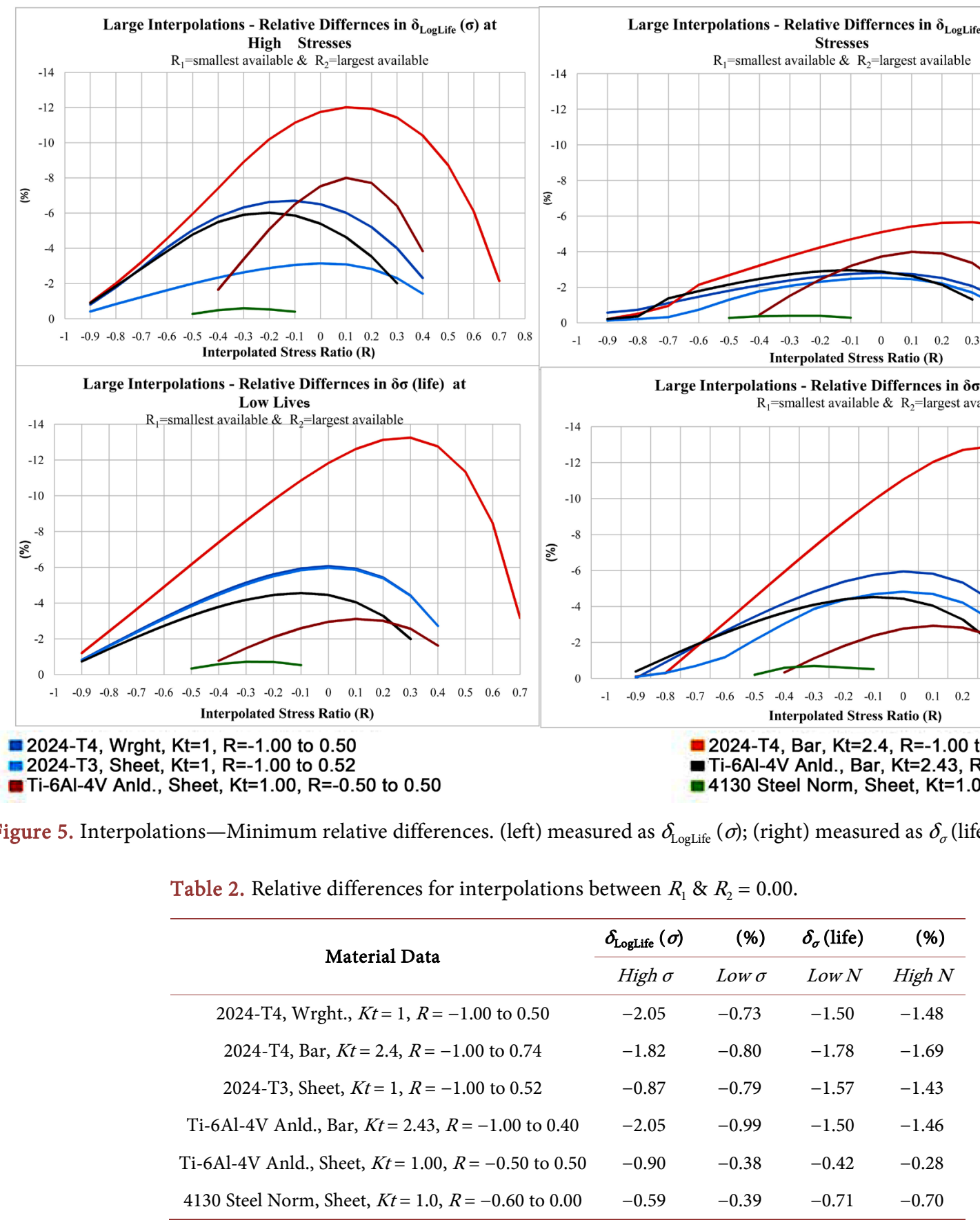

Table 3. Relative differences for interpolations between $R_{1}=0.00 \& R_{2}$.

\begin{tabular}{ccccc}
\hline Material Data & $\boldsymbol{\delta}_{\text {LogLife }}(\sigma)$ & $(\%)$ & $\boldsymbol{\delta}_{\sigma}$ (life) & (\%) \\
\cline { 2 - 5 } & High $\sigma$ & Low $\sigma$ & Low $N$ & High $N$ \\
\hline 2024-T4, Wrght., $K t=1, R=-1.00$ to 0.50 & -1.37 & -0.69 & -1.50 & -1.43 \\
2024-T4, Bar, $K t=2.4, R=-1.00$ to 0.74 & -4.07 & -2.24 & -5.20 & -4.91 \\
2024-T3, Sheet, $K \mathrm{t}=1, R=-1.00$ to 0.52 & -0.73 & -0.55 & -1.40 & -1.10 \\
Ti-6Al-4V Anld., Bar, $K t=2.43, R=-1.00$ to 0.40 & -0.87 & -0.53 & -0.80 & -0.80 \\
Ti-6Al-4V Anld., Sheet, $K t=1.00, R=-0.50$ to 0.50 & -2.92 & -1.61 & -1.23 & -1.09 \\
4130 Steel Norm, Sheet, $K t=1.0, R=-0.60$ to 0.00 & - & - & - & - \\
\hline
\end{tabular}


estimations, it is necessary to work with the stresses at the notch established by using non-linear finite element results calculated with material's stress-strain curve or a Ramberg-Osgood type idealization and generate new input $\mathrm{S} / \mathrm{N}$ curves from the known data. After computation, the curves for the new stress ratios may converted back to net or gross area average stress.

Often for high concentration factors in sheet/plate specimens, the $\mathrm{S} / \mathrm{N}$ data available is for curves of constant mean stress not for constant $R$. In these situations the term $R$ for the stress ratio in Equation (4) to (11) has to be replaced with:

$$
\sigma_{\text {mean }}=\frac{\sigma_{\max }}{R-\sigma_{\max }},
$$

and the curves for the notch area derived as described above. In these cases, for uni-axial estimates, principal or von Mises stresses might be more representative.

While the estimates by interpolation of $\mathrm{S} / \mathrm{N}$ curves can be done quite easy graphically or algebraically or, they can be simply avoided at the design stage by using the conservative curve, extrapolations of the same nature are rarely advisable. The algorithm proposed provides a mean for generating conservative $\mathrm{S} / \mathrm{N}$ curves by extrapolation and the verifications conducted enable a quantifiable estimate of maximum errors. For predictions of fatigue performance the normal safety factors are required to address the scatter in fatigue test results.

\section{Summary}

The aim of this study was to develop a method that enables quick evaluation of the duration for crack initiation during the design stage of parts expected to see loading spectra with multiple amplitude and mean values. For this purpose a method for generating $\mathrm{S} / \mathrm{N}$ curves for multiple stress ratios by interpolation or extrapolation from the data available in two such curves was developed and tested. Generation of similar curves for multiple mean stresses was discussed in the context of notches with high concentration factors. Combining this procedure with Miner's rule and the knock down factors specific to particular applications, durations for crack initiation can be estimated for spectra with many loads. The procedure proposed has the following characteristics:

- Is based entirely on net or gross area stresses which can be measured or derived conveniently;

- Is applicable to cycles with bulk stresses in elastic and/or plastic regime;

- Is working with digitized curves;

- Provides results of a quantifiable precision, conservative in all cases of extrapolations and slightly optimistic for interpolations;

- Together with statistically based methods (like the staircase method), this procedure is able to provide testing laboratories with alternatives to physical testing when developing stress based life curves; 


\section{Acknowledgements}

Many thanks to my son Michael for his assistance in with the research work and consultations and to Steve Swift for advice and telephone consultations during this project.

\section{References}

[1] Peridas, G., Korsunsky, A.M. and Hills, D.A. (2003) The Relationship between the Dang Van Criterion and the Traditional Bulk Fatigue.

http://journals.sagepub.com/doi/abs/10.1243/030932403765310536?journalCode=sd 迆

[2] LexTech Inc. (2018) AFGROW Verion 5.03.01.21-Users Guide and Technical Manual.

[3] Suresh, S. (1998) Fatigue of Materials. 2nd Edition, Cambridge University Press.

[4] Dang Van, K., Griveau, B. and Message, O. On a New Multiaxial Fatigue Limit Criterion: Theory and Application. Biaxial and Multiaxial Fatigue EGF3. In: Brown, M.W. and Miller, K.J., Eds., International Conference on Biaxiall Multiaxial Fatigue (2nd: 1985: University of Sheffield) Mechanical Engineering Publications, London, 459-478.

[5] Dang Van, K., Cailletaud, G., Flavenot, J.F., Le Douaron, A. and Lieurade, H.P. Criterion for High Cycle Fatigue Failure under Multiaxial Loading. Biaxial and Multiaxial Fatigue EGF3. In: Brown, M.W. and Miller, K.J., Eds., International Conference on Biaxiall Multiaxial Fatigue (2nd: 1985: University of Sheffield) Mechanical Engineering Publications, London, 479-496.

[6] Dang Van, K., Le Douaron, A. and Lieurade H.P. Criterion for High Multiaxial Fatigue Limit: A New Approach.

https://www.sciencedirect.com/science/article/pii/B978148328440850185X http://www.gruppofrattura.it/ocs/index.php/ICF/ICF6/paper/viewFile/3155/8217

[7] Charkaluk, E., Constantinescu, A., Maitournam, H. and Dang Van, K. Revisiting the Dang Van Criterion.

https://www.sciencedirect.com/science/article/pii/S1877705809000344

[8] Federal Aviation Administration-Metallic Materials Properties Development and Standardization, Version 7 (MMPDS-7).

http://www.worldcat.org/title/mmpds-07-metallic-materials-properties-developmen t-and-standardization-mmpds/oclc/822046630 


\section{Notations}

a real constant

$b \quad$ real constant

$\mathrm{ksi} \quad \mathrm{kilo}$ pound $/ \mathrm{in}^{2},(1 \mathrm{ksi}=6.895 \mathrm{MPa})$

$t \quad$ time

$A \quad$ amplitude of cyclic stresses

$D, D^{\prime} \quad$ lines representing crack initiation domain for unnotched specimens/ faraway indicators for crack initiation in notched specimens

$F_{t u} / F_{c u} \quad$ ultimate tensile/compressive stress

$F_{t y} / F_{c y} \quad$ yield tensile/compressive stress

$L \quad$ material longitudinal direction

LT material long transversal direction

$N_{f} \quad$ number of cycles to fatigue

$R \quad$ cycle stress ratio $R=\sigma_{\min } / \sigma_{\max }$

$R_{1}, R_{2} \quad$ stress ratios of the known best-fit curves

$\delta R \quad R_{2}-R_{1}\left(R_{2}>R_{1}\right)$, distance from $R$ to the closest known curve

$\tau \quad$ microscopic shear stress

$\underline{\sigma} \quad$ microscopic stress tensor

$\sigma_{\min } \quad$ minimum cycle stress (macroscopic)

$\sigma_{\max } \quad$ maximum cycle stress (macroscopic)

$\sigma_{\text {mean }} \quad$ mean cycle stress $\left(\sigma_{\max }+\sigma_{\min }\right) / 2$

$\Sigma \quad$ macroscopic stress tensor

$\omega$ pulsation

ph microscopic hydrostatic pressure

$P H \quad$ macroscopic hydrostatic pressure

$\underline{s}$ microscopic deviatoric stress tensor

$\underline{S}$ macroscopic deviatoric stress tensor

$q^{*} \quad$ deviatoric component of the stabilized microscopic residual stress tensor

Anld. annealed form

Norm. normalized form

Wrght. Wrought form (drawn, rolled or extruded) 